\title{
Cerebrospinal Fluid of Preeclamptic and Normotensive Pregnant Women Compared to Nonpregnant Women Analyzed with Mass Spectrometry
}

\author{
Coşkun Güzel, Caroline B. van den Berg, Seppe Koopman, Robbert Jan van Krugten, Marcel Stoop, \\ Christoph Stingl, Johannes J. Duvekot, and Theo M. Luider*
}

Cite This: ACS Omega 2020, 5, 32256-32266

Read Online

ACCESS | Lلll Metrics \& More | 国 Article Recommendations | (1) Supporting Information

ABSTRACT: Preeclampsia is a pregnancy-specific multiorgan disorder in which impaired placental functioning and excessive oxidative stress play an important role. We previously showed distinct differences between cerebrospinal fluid proteins in patients with preeclampsia and normotensive pregnant women. An additional group of nonpregnant women was included to study the presence of pregnancy-related proteins in normotensive and preeclamptic pregnancies and whether pregnancy-related proteins were associated with preeclampsia. Cerebrospinal fluid samples were tryptically digested and subsequently measured with a nanoLC-tribrid Orbitrap mass spectrometry system. Proteins were identified by shotgun proteomic analysis based on a data-dependent acquisition method. Proteins identified in preeclampsia, normotensive pregnant controls, and nonpregnant groups were compared to the Progenesis method according to the criteria as previously described and with a secondary analysis using a Scaffold method including Benjamini-Hochberg correction for multiple testing. For preeclampsia, the Progenesis and the Scaffold method together identified 15 (eight proteins

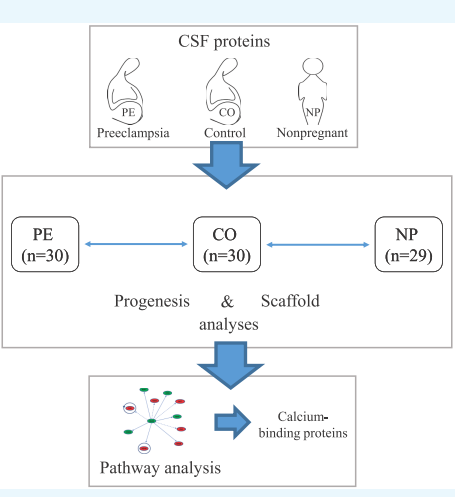
for both analyses with one overlap) proteins that were significantly different compared to normotensive control pregnancies. Three of these 15 proteins, which were elevated in cerebrospinal fluid of preeclamptic women, were described to be pregnancy proteins with a calcium-binding function. Using two analysis methods (Progenesis and Scaffold), four out of 15 differential proteins were associated with pregnancy, as described in the literature. Three out of the four pregnancy-related proteins were elevated in preeclampsia. Furthermore, the contribution of elevated $(n=4 / 15)$ and downregulated $(n=2 / 15)$ calcium-binding proteins in preeclampsia is remarkably high $(40 \%)$ and needs to be elucidated further.

\section{INTRODUCTION}

Preeclampsia (PE) is a major cause of maternal and fetal morbidity and mortality. ${ }^{1}$ Two to eight percent of all pregnancies are complicated by PE worldwide. The maternal brain may be affected in severe PE, which might lead to major cerebrovascular complications such as eclampsia and stroke. ${ }^{2-4}$ The exact biological pathway of PE and its complications in the maternal brain have not been elucidated yet. These complications may result from a disturbed autoregulatory response of the brain to increased blood pressure and generalized endothelial cell dysfunction. Endothelial cells play a crucial role in the homeostatic regulation and stability of the brain microenvironment. ${ }^{5,6}$ The endothelial cells of the bloodbrain barrier (BBB) are coupled by tight and adhered junctions adhered to a basement membrane reducing paracellular diffusion, preventing some blood constituents from passing into the brain's extracellular space. ${ }^{7}$ The $\mathrm{BBB}$ is a selective barrier that controls the transport of molecules between the vascular system and brain. ${ }^{8}$ Inflammatory cells and selected proteins can penetrate the brain when the BBB is dysregulated, causing immunological reactions, including edema and cell death. ${ }^{, 10} \mathrm{PE}$ is suggested to increase the BBB permeability in an animal model, ${ }^{11}$ although this is not seen in preeclamptic women. ${ }^{12}$

Cerebrospinal fluid (CSF), including proteins, surrounds the brain and is partly produced in the brain by the choroid plexus and partly in ependyma. The protein concentration in CSF is approximately 100-200 times lower than in serum, which enables easier detection of lower abundant proteins, and it is assumed to be an approach to identify brain-derived proteins that relate to brain pathology during the patient's life. ${ }^{13,14} \mathrm{CSF}$ is in contact with neuronal tissue and will reflect to a certain extent, the surrounding neuronal tissue content. ${ }^{15,16}$ Therefore, investigating CSF might be useful to gain more insight into brain pathophysiology during pregnancies complicated by PE. A proteomic study that describes an overview of pregnancy

Received: August 13, 2020

Accepted: November 13, 2020

Published: December 10, 2020 
Scheme 1. Flowchart of the Current Study in Comparison to the Previous Study

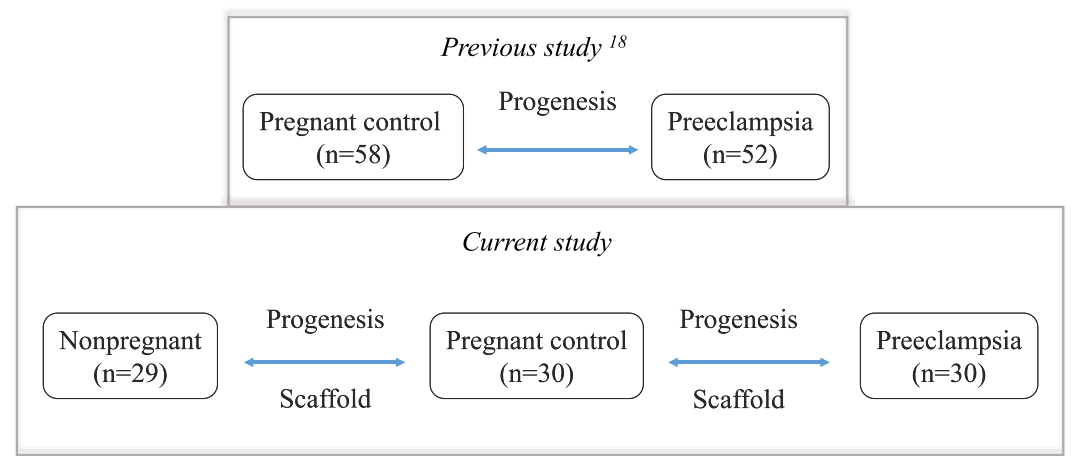

proteins in CSF has not been described yet in the literature. Also, the exact functions of these large numbers of pregnancy proteins on brain function are still unknown. ${ }^{17}$ We previously showed distinct differences between CSF proteins in patients with $\mathrm{PE}$ and normotensive pregnant women. From these proteins, AMBP was the most significant one. ${ }^{18}$ In the present study, we included a nonpregnant control group to compare also proteins in CSF between normotensive pregnant women and nonpregnant women by shotgun proteomics technology. In this way, we were able to study the effects of pregnancy on the composition of CSF proteins in normotensive and preeclamptic pregnancies.

\section{RESULTS}

The general patient characteristics are shown in Table S1, clinical characteristics. Maternal age was not significantly different in preeclamptic compared to normotensive pregnant and nonpregnant women. Nonpregnant women smoked more frequently compared to pregnant participants. Gestational age at sampling and birth weight was lower in the $\mathrm{PE}$ group compared to the pregnant women. The total protein abundances (UV traces) in CSF between the three groups were not significantly different, although a higher trend was observed in nonpregnant women. Comparisons between preeclamptic, normotensive, and nonpregnant women groups were analyzed with the Progenesis and Scaffold method and a part of the results compared to the previous study, ${ }^{18}$ as illustrated in Scheme 1.

Pregnancy-Related Proteins in CSF. For protein identifications, a shotgun proteomic analysis was performed for each CSF sample from the preeclamptic, normotensive pregnant, and nonpregnant women. The number of total identified CSF proteins was 798, 802, and 746, respectively, per group.

Significantly different proteins related to pregnancy were identified through both methods, Progenesis $(n=23)$ and Scaffold $(n=135)$. Pregnancy proteins mentioned in the Scopus database were found by comparing normotensive pregnant women with nonpregnant women. Both up- and downregulated CSF proteins were found, as shown in Tables 1 and 2 for both methods, respectively.

When comparing normotensive pregnant women with nonpregnant women, a $7 \%$ higher number of proteins were identified in pregnant women. We observed that most of the significantly different proteins analyzed by the methods Progenesis $(n=20)$ and Scaffold $(n=81)$ were upregulated in normotensive pregnant women, and, through IPA, that most of the proteins are enzymes and might have a function in protein synthesis (metabolism of proteins).

CSF Proteins in Preeclamptic Women Compared to Normotensive Pregnant Women. Using the Progenesis method, a total of 155 peptides were identified that were significantly different $(p<0.01)$ in CSF between preeclamptic and normotensive women; with a permutation test $(n=1000)$, a background of $67 \pm 30$ was observed. The significant peptides correlated to eight proteins that passed the criteria. These proteins were considered as differentially abundant between preeclamptic patients and normotensive pregnant women. Four of these CSF proteins showed a higher abundance in PE (Table 3).

Scaffold analysis between the preeclamptic group and the normotensive pregnant group showed eight proteins, which were significantly different after applying Benjamini-Hochberg analysis correcting for multiple testing (Table 4).

Matrix GLA protein (MGP) was identified by both the Progenesis method and the Scaffold method. We did not find significant differences in the number of total proteins identified in CSF of preeclamptic women compared to normotensive pregnant women, 798 and 802 , respectively.

For the comparison of CSF proteins in women with PE and normotensive pregnant women, as also previously described, ${ }^{18}$ 510 peptides $(p<0.01)$ corresponding to 17 significant proteins were identified using only the Progenesis method. The permutation test gave a range of $67 \pm 28$ peptides. Three of the 17 significant proteins were higher represented in nonpregnant women.

All peptides of the protein AMBP, analyzed as the most significant differentially abundant protein in the previous paper, ${ }^{18}$ were more abundant in preeclamptic women than in the normotensive pregnant women $(p=0.041)$. However, the protein did not pass the criteria for significance analyzed by the Progenesis method in the present study. Although there was no overlap with the significant differentially abundant proteins in CSF of preeclamptic patients listed in Table 2 of the previous work, ${ }^{18}$ all proteins (except one) mentioned in Table 2 showed the same trends (i.e., up- or downregulated) in this study using the Progenesis method. Moreover, the protein Amyloid-like protein 1 was not found in the present study, but the protein isoform Amyloid-like protein 2 instead (Table S4, Supporting Information).

By the Progenesis and Scaffold methods, MGP passed the criteria for significance and was upregulated in PE. MGP was also significantly higher in preeclamptic women compared to normotensive pregnant controls $(p<0.00010$, BenjaminiHochberg) when analyzed by the Scaffold method (Figure 1). 


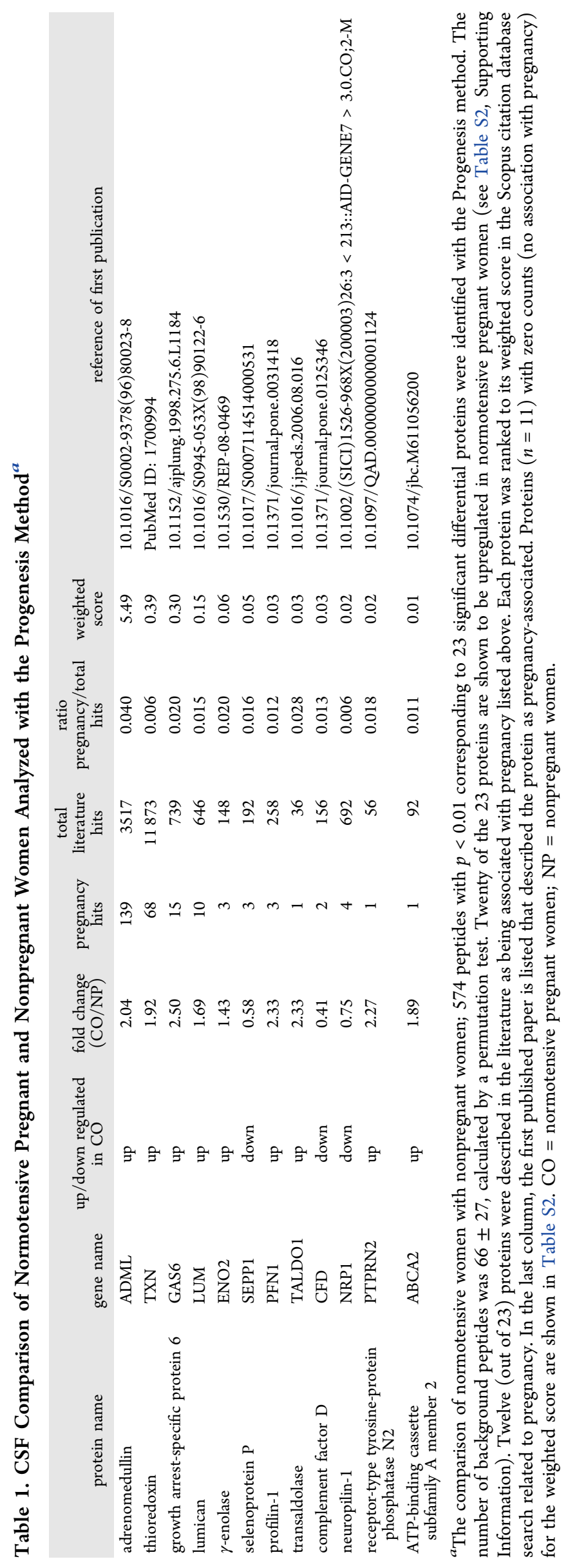


Table 2. CSF Protein Comparison of Normotensive Pregnant and Nonpregnant Women Analyzed with the Scaffold Method ${ }^{a, b}$

\begin{tabular}{|c|c|c|c|c|c|c|c|c|c|}
\hline protein name & gene name & $t$-test & $\begin{array}{l}\text { up/ } \\
\text { down } \\
\text { in CO }\end{array}$ & $\begin{array}{l}\text { fold } \\
\text { change } \\
(\mathrm{CO} / \\
\mathrm{NP})\end{array}$ & $\begin{array}{l}\text { pregnancy } \\
\text { hits }\end{array}$ & $\begin{array}{l}\text { total } \\
\text { literature } \\
\text { hits }\end{array}$ & $\begin{array}{l}\text { ratio } \\
\text { pregnancy/ } \\
\text { total hits }\end{array}$ & $\begin{array}{l}\text { weighted } \\
\text { score }\end{array}$ & reference of first publication \\
\hline prolactin & PRL & $\begin{array}{r}2.50 \times \\
10^{-3}\end{array}$ & up & 2.79 & 3624 & 48741 & 0.07 & 269.45 & $10.1210 /$ endo-17-6-689 \\
\hline pregnancy zone protein & PZP & $\begin{array}{l}<1.00 \times \\
10^{-4}\end{array}$ & up & 1.74 & 197 & 197 & 1.00 & 197.00 & $\begin{array}{l}10.1016 / 0002-9378(64) \\
90712-4\end{array}$ \\
\hline fibronectin & FN1 & $\begin{array}{r}2.20 \times \\
10^{-3}\end{array}$ & down & 0.91 & 643 & 4640 & 0.14 & 89.11 & Kuusela et al., 1978 \\
\hline pregnancy-specific $\beta$-1-glycoprotein 1 & PSG1 & $\begin{array}{c}<1.00 \times \\
10^{-4}\end{array}$ & up & 43.41 & 34 & 62 & 0.55 & 18.65 & $\begin{array}{l}\text { 10.1016/0006-291X(89) } \\
\text { 91105-4 }\end{array}$ \\
\hline sex hormone-binding globulin & SHBG & $<1.00 \times$ & up & 15.56 & 273 & 5300 & 0.05 & 14.06 & Rager et al., 1972 \\
\hline $\begin{array}{l}\text { disintegrin and metalloproteinase } \\
\text { domain-containing protein } 12\end{array}$ & ADAM12 & $\begin{array}{r}2.60 \times \\
10^{-3}\end{array}$ & up & 4.44 & 67 & 357 & 0.19 & 12.57 & $10.1002 /$ pd.762 \\
\hline pregnancy-specific $\beta$-1-glycoprotein 9 & PSG9 & $\begin{array}{c}<1.00 \times \\
10^{-4}\end{array}$ & up & 21.26 & 13 & 14 & 0.93 & 12.07 & 10.1021/bi00496a009 \\
\hline $\begin{array}{l}\text { pregnancy-specific } \beta \text {-1-glycoprotein } \\
\quad 11\end{array}$ & PSG11 & $\begin{array}{c}<1.00 \times \\
10^{-4}\end{array}$ & up & 11.88 & 11 & 16 & 0.69 & 7.56 & Kuroki et al., 1991 \\
\hline ceruloplasmin & $\mathrm{CP}$ & $\begin{array}{r}2.50 \times \\
10^{-3}\end{array}$ & up & 1.16 & 221 & 7444 & 0.03 & 6.56 & $10.3181 / 00379727-98-24117$ \\
\hline pregnancy-specific $\beta$-1-glycoprotein 4 & PSG4 & $\begin{array}{c}<1.00 \times \\
10^{-4}\end{array}$ & up & 17.27 & 9 & 15 & 0.60 & 5.40 & $\begin{array}{l}\text { 10.1016/0006-291X(89) } \\
91105-4\end{array}$ \\
\hline $\begin{array}{l}\text { chorionic somatomammotropin } \\
\text { hormone } 1\end{array}$ & $\mathrm{CSH} 1$ & $\begin{array}{c}<1.00 \times \\
10^{-4}\end{array}$ & up & 81.57 & 21 & 90 & 0.23 & 4.90 & $10.1373 / 49.9 .1445$ \\
\hline pregnancy-specific $\beta$-1-glycoprotein 3 & PSG3 & $\begin{array}{c}<1.00 \times \\
10^{-4}\end{array}$ & up & 16.56 & 11 & 26 & 0.42 & 4.65 & $\begin{array}{l}10.1016 / 0006-291 X(90) \\
91455-2\end{array}$ \\
\hline $\begin{array}{l}\text { putative pregnancy-specific } \\
\beta \text {-1-glycoprotein } 7\end{array}$ & PSG7 & $\begin{array}{c}<1.00 \times \\
10^{-4}\end{array}$ & up & 21.41 & 6 & 9 & 0.67 & 4.00 & $\begin{array}{l}\text { 10.1016/0006-291X(90) } \\
\text { 92103-7 }\end{array}$ \\
\hline serum albumin & ALB & $<1.00 \times$ & down & 0.81 & 540 & 86597 & 0.01 & 3.37 & $\begin{array}{l}\text { 10.1111/j.0954-6820.1921. } \\
\text { tb15205.x }\end{array}$ \\
\hline vitamin $\mathrm{D}$ binding protein & GC & $\begin{array}{r}9.00 \times \\
10^{-4}\end{array}$ & up & 1.14 & 62 & 1731 & 0.04 & 2.22 & $10.1210 /$ jcem-45-2-225 \\
\hline $72 \mathrm{kDa}$ type IV collagenase & MMP2 & $\begin{array}{c}<1.00 \times \\
10^{-4}\end{array}$ & up & 1.43 & 90 & 4325 & 0.02 & 1.87 & PubMed ID: 11042903 \\
\hline $\begin{array}{l}\text { insulin-like growth factor-binding } \\
\text { protein } 3\end{array}$ & IGFBP3 & $\begin{array}{r}8.30 \times \\
10-3\end{array}$ & up & 1.80 & 32 & 1128 & 0.03 & 0.91 & 10.1007/BF02211374 \\
\hline pregnancy-specific $\beta$-1-glycoprotein 2 & PSG2 & $\begin{array}{c}<1.00 \times \\
10^{-4}\end{array}$ & up & 20.53 & 6 & 40 & 0.15 & 0.90 & $\begin{array}{l}\text { 10.1016/0006-291X(90) } \\
91455-2\end{array}$ \\
\hline afamin & AFM & $\begin{array}{r}6.00 \times \\
10^{-4}\end{array}$ & up & 1.34 & 9 & 96 & 0.09 & 0.84 & $10.1002 / \mathrm{pd} .2040$ \\
\hline glutathione peroxidase 3 & GPX3 & $\begin{array}{l}<1.00 \times \\
10^{-4}\end{array}$ & up & 2.73 & 20 & 488 & 0.04 & 0.82 & Sunde et al., 1998 \\
\hline$\beta$-2-microglobulin & $\mathrm{B} 2 \mathrm{M}$ & $\begin{array}{r}7.00 \times \\
10^{-4}\end{array}$ & up & 1.23 & 55 & 3794 & 0.01 & 0.80 & $10.3109 / 00365518109090529$ \\
\hline bone marrow proteoglycan & PRG2 & $<1.00 \times$ & up & 5.90 & 5 & 33 & 0.15 & 0.76 & $\begin{array}{l}\text { 10.1095/ } \\
\text { biolreprod.110.090209 }\end{array}$ \\
\hline somatostatin & SST & $\begin{array}{r}2.00 \times \\
10^{-4}\end{array}$ & up & 2.30 & 153 & 32876 & 0.00 & 0.71 & Kumasaka et al., 1978 \\
\hline collagen $\alpha$-1(III) chain & COL3A1 & $\begin{array}{r}4.20 \times \\
10^{-3}\end{array}$ & down & 0.47 & 24 & 890 & 0.03 & 0.65 & $\begin{array}{l}\text { 10.1002/(SICI) } 1096-8628 \\
(19990212) 82: 4<305: \text { AID- } \\
\text { AJMG6>3.0.CO;2-C }\end{array}$ \\
\hline histidine-rich glycoprotein & HRG & $\begin{array}{c}<1.00 \times \\
10^{-4}\end{array}$ & down & 0.33 & 16 & 415 & 0.04 & 0.62 & $10.3181 / 00379727-158-40265$ \\
\hline $\begin{array}{l}\text { insulin-like growth factor-binding } \\
\text { protein } 2\end{array}$ & IGFBP2 & $\begin{array}{c}<1.00 \times \\
10^{-4}\end{array}$ & down & 0.69 & 17 & 505 & 0.03 & 0.57 & $10.1210 /$ endo- $127-5-2270$ \\
\hline glutathione $S$-transferase $\mathrm{P}$ & GSTP1 & $\begin{array}{r}2.10 \times \\
10^{-3}\end{array}$ & up & 1.82 & 43 & 3348 & 0.01 & 0.55 & $10.3109 / 10641959909023074$ \\
\hline apolipoprotein A-I & APOA1 & $\begin{array}{r}2.00 \times \\
10^{-4}\end{array}$ & up & 1.34 & 30 & 1725 & 0.02 & 0.52 & PubMed ID: 8364102 \\
\hline corticosteroid-binding globulin & SERPINA6 & $\begin{array}{r}5.00 \times \\
10^{-4}\end{array}$ & up & 1.56 & 4 & 33 & 0.12 & 0.48 & 10.1002/bdra.20843 \\
\hline $\begin{array}{l}\text { insulin-like growth factor-binding } \\
\text { protein } 5\end{array}$ & IGFBP5 & $\begin{array}{c}<1.00 \times \\
10^{-4}\end{array}$ & down & 0.36 & 13 & 371 & 0.04 & 0.46 & 10.1095/biolreprod62.5.1415 \\
\hline collagen $\alpha-1(\mathrm{I})$ chain & COL1A1 & $\begin{array}{r}4.00 \times \\
10^{-4}\end{array}$ & down & 0.71 & 34 & 2994 & 0.01 & 0.39 & PubMed ID: 2886666 \\
\hline collagen $\alpha-2(\mathrm{I})$ chain & COL1A2 & $\begin{array}{c}<1.00 \times \\
10^{-4}\end{array}$ & down & 0.71 & 21 & 1270 & 0.02 & 0.35 & PubMed ID: 2886666 \\
\hline$\alpha$-1-antitrypsin & SERPINA1 & $\begin{array}{c}<1.00 \times \\
10^{-4}\end{array}$ & up & 1.49 & 11 & 349 & 0.03 & 0.35 & 10.1016/j.ajog.2008.07.006 \\
\hline endothelial protein $\mathrm{C}$ receptor & PROCR & $\begin{array}{r}1.30 \times \\
10^{-3}\end{array}$ & up & 3.20 & 5 & 82 & 0.06 & 0.30 & 10.1160/TH-09-04-0224 \\
\hline
\end{tabular}


Table 2. continued

\begin{tabular}{|c|c|c|c|c|c|c|c|c|c|}
\hline protein name & gene name & $t$-test & $\begin{array}{l}\text { up/ } \\
\text { down } \\
\text { in CO }\end{array}$ & $\begin{array}{l}\text { fold } \\
\text { change } \\
\text { (CO/ } \\
\mathrm{NP})\end{array}$ & $\begin{array}{l}\text { pregnancy } \\
\text { hits }\end{array}$ & $\begin{array}{l}\text { total } \\
\text { literature } \\
\text { hits }\end{array}$ & $\begin{array}{l}\text { ratio } \\
\text { pregnancy/ } \\
\text { total hits }\end{array}$ & $\begin{array}{l}\text { weighted } \\
\text { score }\end{array}$ & reference of first publication \\
\hline growth arrest-specific protein 6 & GAS6 & $\begin{array}{r}1.40 \times \\
10^{-3}\end{array}$ & up & 2.55 & 15 & 750 & 0.02 & 0.30 & $\begin{array}{l}\text { 10.1152/ajplung.1998.275.6. } \\
\text { L1184 }\end{array}$ \\
\hline transgelin & TAGLN & $\begin{array}{l}<1.00 \times \\
10^{-4}\end{array}$ & up & 3.56 & 6 & 143 & 0.04 & 0.25 & 10.1530/REP-09-0208 \\
\hline apolipoprotein E & APOE & $\begin{array}{l}<1.00 \times \\
10^{-4}\end{array}$ & up & 1.27 & 69 & 19608 & 0.00 & 0.24 & PubMed ID: 8725150 \\
\hline clusterin & CLU & $\begin{array}{l}<1.00 \times \\
10^{-4}\end{array}$ & down & 0.80 & 22 & 2311 & 0.01 & 0.21 & 10.1210/endo.136.12.7588296 \\
\hline serotransferrin & $\mathrm{TF}$ & $\begin{array}{l}<1.00 \times \\
10^{-4}\end{array}$ & up & 1.16 & 7 & 253 & 0.03 & 0.19 & $10.1042 / b j 2570231$ \\
\hline $\begin{array}{l}\text { V-set and immunoglobulin domain- } \\
\text { containing protein } 4\end{array}$ & VSIG4 & $\begin{array}{r}6.00 \times \\
10^{-4}\end{array}$ & up & 3.66 & 3 & 55 & 0.05 & 0.16 & $\begin{array}{l}10.3321 / j . i s s n: 0529- \\
567 x .2008 .09 .004\end{array}$ \\
\hline adipocyte enhancer-binding protein 1 & AEBP1 & $\begin{array}{l}<1.00 \times \\
10^{-4}\end{array}$ & down & 0.20 & 3 & 64 & 0.05 & 0.14 & 10.1371/journal.pone.0027795 \\
\hline $\begin{array}{l}\text { lymphatic vessel endothelial } \\
\text { hyaluronic acid receptor } 1\end{array}$ & LYVE1 & $\begin{array}{r}1.80 \times \\
10^{-3}\end{array}$ & up & 1.56 & 4 & 115 & 0.03 & 0.14 & $\begin{array}{l}\text { 10.1095/ } \\
\quad \text { biolreprod.108.067975 }\end{array}$ \\
\hline $\begin{array}{l}\text { extracellular superoxide dismutase } \\
{[\mathrm{Cu}-\mathrm{Zn}]}\end{array}$ & SOD3 & $\begin{array}{r}5.40 \times \\
10^{-3}\end{array}$ & down & 0.85 & 8 & 487 & 0.02 & 0.13 & $\begin{array}{l}10.1016 / \mathrm{j} . \\
\text { placenta.2010.02.007 }\end{array}$ \\
\hline $\begin{array}{l}\text { ectonucleotide pyrophosphatase/ } \\
\text { phosphodiesterase family } \\
\text { member } 2\end{array}$ & ENPP2 & $\begin{array}{r}1.40 \times \\
10^{-3}\end{array}$ & down & 0.86 & 4 & 123 & 0.03 & 0.13 & $\begin{array}{l}\text { 10.1095/ } \\
\text { biolreprod.112.099564 }\end{array}$ \\
\hline renin receptor & ATP6AP2 & $\begin{array}{l}<1.00 \times \\
10^{-4}\end{array}$ & down & 0.36 & 3 & 73 & 0.04 & 0.12 & $\begin{array}{l}\text { 10.1016/j. } \\
\text { placenta.2014.09.004 }\end{array}$ \\
\hline 14-3-3 protein zeta/delta & YWHAZ & $\begin{array}{l}<1.00 \times \\
10^{-4}\end{array}$ & up & 2.19 & 6 & 349 & 0.02 & 0.10 & $\begin{array}{l}\text { 10.1016/j. } \\
\text { placenta.2004.09.009 }\end{array}$ \\
\hline fibulin-2 & FBLN2 & $\begin{array}{r}4.00 \times \\
10^{-3}\end{array}$ & down & 0.58 & 2 & 39 & 0.05 & 0.10 & $10.1007 / \mathrm{s} 00018-014-1577-4$ \\
\hline pleiotrophin & PTN & $\begin{array}{c}<1.00 \times \\
10^{-4}\end{array}$ & down & 0.17 & 8 & 627 & 0.01 & 0.10 & 10.1210/endo.142.2.8111 \\
\hline $\begin{array}{l}\text { WAP four-disulfide core domain } \\
\text { protein } 2\end{array}$ & WFDC2 & $\begin{array}{r}3.70 \times \\
10^{-3}\end{array}$ & up & 2.38 & 2 & 40 & 0.05 & 0.10 & $\begin{array}{l}\text { 10.1111/j.1525- } \\
142 X .2007 .00175 . x\end{array}$ \\
\hline $\begin{array}{l}\text { inter- } \alpha \text {-trypsin inhibitor heavy } \\
\text { chain H4 }\end{array}$ & ITIH4 & $\begin{array}{r}1.60 \times \\
10^{-3}\end{array}$ & up & 1.27 & 3 & 122 & 0.02 & 0.07 & 10.1530/rep.0.1260621 \\
\hline interleukin- 6 receptor subunit $\beta$ & IL6ST & $\begin{array}{r}4.30 \times \\
10^{-3}\end{array}$ & up & 1.75 & 3 & 154 & 0.02 & 0.06 & 10.1530/REP-08-0437 \\
\hline$\beta$-2-glycoprotein 1 & $\mathrm{APOH}$ & $\begin{array}{l}<1.00 \times \\
10^{-4}\end{array}$ & down & 0.82 & 3 & 166 & 0.02 & 0.05 & $\begin{array}{l}\text { 10.1016/j. } \\
\text { earlhumdev.2010.06.013 }\end{array}$ \\
\hline $\begin{array}{l}\text { polypeptide } \\
N \text {-acetylgalactosaminyltransferase } 2\end{array}$ & GALNT2 & $\begin{array}{l}<1.00 \times \\
10^{-4}\end{array}$ & up & 3.41 & 2 & 77 & 0.03 & 0.05 & 10.1289/ehp.1307892 \\
\hline cadherin-18 & CDH18 & $\begin{array}{r}1.10 \times \\
10^{-3}\end{array}$ & up & 1.45 & 1 & 20 & 0.05 & 0.05 & 10.1016/j.tjog.2018.08.023 \\
\hline L-lactate dehydrogenase A chain & LDHA & $\begin{array}{r}8.20 \times \\
10^{-3}\end{array}$ & up & 2.36 & 7 & 1008 & 0.01 & 0.05 & $\begin{array}{l}\text { 10.1016/j. } \\
\text { placenta.2006.11.011 }\end{array}$ \\
\hline $\begin{array}{l}\text { insulin-like growth factor-binding } \\
\text { protein } 7\end{array}$ & IGFBP7 & $<1.00 \times$ & down & 0.70 & 4 & 364 & 0.01 & 0.04 & 10.1371/journal.pone. 0045224 \\
\hline peptidyl-prolyl cis-trans isomerase A & PPIA & $\begin{array}{r}2.00 \times \\
10^{-4}\end{array}$ & up & 1.97 & 4 & 424 & 0.01 & 0.04 & $10.1186 / 1471-2199-10-100$ \\
\hline heat shock cognate $71 \mathrm{kDa}$ protein & HSPA8 & $\begin{array}{r}3.20 \times \\
10^{-3}\end{array}$ & up & 1.85 & 3 & 246 & 0.01 & 0.04 & $10.1007 / \mathrm{s} 11033-012-2104-\mathrm{z}$ \\
\hline profilin-1 & PFN1 & $\begin{array}{r}2.00 \times \\
10^{-4}\end{array}$ & up & 3.03 & 3 & 258 & 0.01 & 0.03 & 10.1371/journal.pone. 0031418 \\
\hline glutaminyl-peptide cyclotransferase & QPCT & $\begin{array}{r}1.30 \times \\
10^{-3}\end{array}$ & up & 3.53 & 1 & 34 & 0.03 & 0.03 & $10.3390 /$ nu 10040417 \\
\hline $\begin{array}{l}\text { cocaine- and amphetamine-regulated } \\
\text { transcript protein }\end{array}$ & CARTPT & $\begin{array}{r}2.30 \times \\
10^{-3}\end{array}$ & up & 2.23 & 1 & 36 & 0.03 & 0.03 & 10.1038/ijo.2015.38 \\
\hline $\begin{array}{l}\text { procollagen C-endopeptidase } \\
\text { enhancer } 1\end{array}$ & PCOLCE & $\begin{array}{l}<1.00 \times \\
10^{-4}\end{array}$ & down & 0.26 & 1 & 36 & 0.03 & 0.03 & $10.1089 / \mathrm{scd} .2013 .0334$ \\
\hline complement factor D & CFD & $\begin{array}{l}<1.00 \times \\
10^{-4}\end{array}$ & down & 0.38 & 2 & 156 & 0.01 & 0.03 & 10.1371/journal.pone. 0125346 \\
\hline $\begin{array}{l}\text { peptidyl-glycine } \alpha \text {-amidating } \\
\text { monooxygenase }\end{array}$ & PAM & $\begin{array}{r}4.90 \times \\
10^{-3}\end{array}$ & up & 1.24 & 1 & 45 & 0.02 & 0.02 & $\begin{array}{l}\text { 10.1016/j. } \\
\text { theriogenology.2016.10.007 }\end{array}$ \\
\hline apolipoprotein A-II & APOA2 & $\begin{array}{r}1.80 \times \\
10^{-3}\end{array}$ & up & 1.46 & 2 & 188 & 0.01 & 0.02 & $10.1262 /$ jrd.18002 \\
\hline matrix gla protein & MGP & $\begin{array}{r}3.60 \times \\
10^{-3}\end{array}$ & down & 0.42 & 4 & 863 & 0.00 & 0.02 & $\begin{array}{l}\text { 10.1111/j.1834-7819.1994. } \\
\text { tb01379.x }\end{array}$ \\
\hline$\alpha$-enolase & ENO1 & $\begin{array}{l}<1.00 \times \\
10^{-4}\end{array}$ & up & 2.68 & 4 & 922 & 0.00 & 0.02 & 10.1076/apab.110.1.94.897 \\
\hline $\begin{array}{l}\text { cadherin EGF LAG seven-pass G- } \\
\text { type receptor } 2\end{array}$ & CELSR2 & $\begin{array}{l}<1.00 \times \\
10^{-4}\end{array}$ & down & 0.30 & 1 & 87 & 0.01 & 0.01 & $10.3389 /$ fgene. 2019.00540 \\
\hline
\end{tabular}


Table 2. continued

\begin{tabular}{|c|c|c|c|c|c|c|c|c|c|}
\hline protein name & gene name & $t$-test & $\begin{array}{l}\text { up/ } \\
\text { down } \\
\text { in CO }\end{array}$ & $\begin{array}{c}\text { fold } \\
\text { change } \\
(\mathrm{CO} / \\
\mathrm{NP})\end{array}$ & $\begin{array}{l}\text { pregnancy } \\
\text { hits }\end{array}$ & $\begin{array}{c}\text { total } \\
\text { literature } \\
\text { hits }\end{array}$ & $\begin{array}{c}\text { ratio } \\
\text { pregnancy/ } \\
\text { total hits }\end{array}$ & $\begin{array}{l}\text { weighted } \\
\text { score }\end{array}$ & reference of first publication \\
\hline spondin-1 & SPON1 & $\begin{array}{l}<1.00 \times \\
10^{-4}\end{array}$ & down & 0.23 & 1 & 90 & 0.01 & 0.01 & $10.1071 / \mathrm{RD} 18114$ \\
\hline $\begin{array}{l}\text { ATP-binding cassette subfamily A } \\
\text { member } 2\end{array}$ & $\mathrm{ABCA} 2$ & $\begin{array}{l}<1.00 \times \\
10^{-4}\end{array}$ & up & 5.21 & 1 & 92 & 0.01 & 0.01 & 10.1074/jbc.M611056200 \\
\hline $\begin{array}{l}\text { lysosome-associated membrane } \\
\text { glycoprotein } 2\end{array}$ & LAMP2 & $\begin{array}{r}3.00 \times \\
10^{-4}\end{array}$ & up & 1.79 & 2 & 471 & 0.00 & 0.01 & 10.1016/j.amjcard.2011.09.024 \\
\hline thrombospondin-2 & THBS2 & $\begin{array}{r}2.80 \times \\
10^{-3}\end{array}$ & down & 0.26 & 1 & 161 & 0.01 & 0.01 & $10.1262 /$ jrd.2016-056 \\
\hline basal cell adhesion molecule & BCAM & $\begin{array}{r}4.60 \times \\
10^{-3}\end{array}$ & up & 2.14 & 1 & 176 & 0.01 & 0.01 & $10.3233 / \mathrm{CH}-2011-1525$ \\
\hline
\end{tabular}

${ }^{a} \mathrm{~A}$ reference to the literature was included to define the association of the particular protein in pregnancy. ${ }^{b} \mathrm{~A}$ total number of 135 proteins was found with a $p$-value of less than $p=0.0086$ (Benjamini-Hochberg) when comparing the normotensive pregnant women with nonpregnant women. Seventy-three (out of 135) proteins were described in the literature as being associated with pregnancy listed above. Each protein was ranked to their weighted score in the Scopus citation database search (November 2019) related to pregnancy. In the last column, the first published paper is listed. As an example, prolactin has the highest score for a protein described to associate with pregnancy. Proteins $(n=62)$ with zero counts (no association with pregnancy) for the weighted score are shown in Table S3, Supporting Information. CO = normotensive pregnant women; NP = nonpregnant women.

Table 3. Differently Abundant Proteins $(n=8)$ in CSF of Women with Preeclampsia and Normotensive Pregnant Women, as Analyzed by the Progenesis Method $^{a, b}$

\begin{tabular}{|c|c|c|c|}
\hline protein name & gene name & up/down in PE & fold change (PE/CO) \\
\hline augurin & C2orf 40 & PE & 1.11 \\
\hline coagulation factor $\mathrm{X}^{e}$ & F10 & PE & 1.41 \\
\hline matrix Gla protein ${ }^{c, d, e}$ & MGP & $\mathrm{PE}$ & 2.13 \\
\hline osteomodulin & OMD & $\mathrm{PE}$ & 1.25 \\
\hline cadherin- $4^{e}$ & $\mathrm{CDH} 4$ & $\mathrm{CO}$ & 0.72 \\
\hline podocalyxin-like protein 2 & PODXL2 & $\mathrm{CO}$ & 0.79 \\
\hline receptor-type tyrosine-protein phosphatase F & PTPRF & $\mathrm{CO}$ & 0.65 \\
\hline nectin-1 & PVRL1 & $\mathrm{CO}$ & 0.77 \\
\hline
\end{tabular}

${ }^{a} \mathrm{CO}=$ normotensive pregnant women. ${ }^{b} \mathrm{PE}=$ preeclamptic women. ${ }^{c}$ Significantly different protein as analyzed by Benjamini-Hochberg $(p<$ 0.00055 ) using the Scaffold method. ${ }^{d}$ Related to pregnancy (see Table 1 ). ${ }^{e}$ Calcium-binding protein.

Table 4. Differently Abundant Proteins $(n=8)$ in CSF of Women with Preeclampsia and Normotensive Women as Analyzed by the Scaffold Method ${ }^{a, b}$

\begin{tabular}{llccc}
\multicolumn{1}{c}{ protein name } & gene name & up/down in PE & $t$-test & fold change (PE/CO) \\
actin, $\alpha$ skeletal muscle & ACTA1 & PE & $1.60 \times 10^{-4}$ & 1.48 \\
basement membrane-specific heparan sulfate proteoglycan core protein $^{e}$ & HSPG2 & PE & $<1.00 \times 10^{-4}$ & 1.72 \\
fibromodulin $^{-4}$ & FMOD & PE & $<1.00 \times 10^{-4}$ & 1.57 \\
fibronectin $^{d e}$ & FN1 & PE & $1.40 \times 10^{-4}$ & 1.11 \\
matrix Gla protein $^{c, d, e}$ & MGP & PE & $<1.00 \times 10^{-4}$ & 3.80 \\
transgelin $^{d, e}$ & TAGLN & PE & $<1.00 \times 10^{-4}$ & 2.98 \\
chromogranin-A $^{-4}$ & CHGA & CO & $<1.00 \times 10^{-4}$ & 0.77 \\
peptidyl-glycine $\alpha$-amidating monooxygenase $^{d}$ & PAM & CO & $4.50 \times 10^{-4}$ & 0.77
\end{tabular}

${ }^{a} \mathrm{CO}=$ normotensive pregnant women. ${ }^{b} \mathrm{PE}=$ preeclamptic women. ${ }^{c}$ Significantly different protein as analyzed by the Progenesis method. ${ }^{d}$ Related to pregnancy (see Table 2 ). ${ }^{e}$ Calcium-binding protein.

The principal component analysis (PCA) for the comparison of the preeclamptic, normotensive pregnant, and nonpregnant women is shown in Figure 2. As expected, the nonpregnant women (indicated in green) could be separated from the PE (indicated in red) and normotensive pregnant women (indicated in blue) groups. The preeclamptic women clustered with the normotensive pregnant women, indicating that there is no large difference between these two groups that can be visualized directly by this PCA approach.

\section{DISCUSSION}

The analysis of CSF proteins in normotensive pregnant women compared to nonpregnant women has not been described previously. In CSF of normotensive pregnant women, significantly different peptides and proteins were found to be associated with pregnancy compared to nonpregnant women using two methods (Progenesis method $(n=12)$ and Scaffold method $(n=73))$. The overlap of both methods based on peptide and protein levels was low. The Progenesis method is very restrictive, which may lead to a high rate of false negatives. Using the Scaffold method, which includes BenjaminiHochberg correction for multiple testing, shows that the rate of false negatives is low. The proteins identified by both methods complement each other because one approach is more focused on peptides (Progenesis method), and the other approach (Scaffold method) is more focused on proteins. 


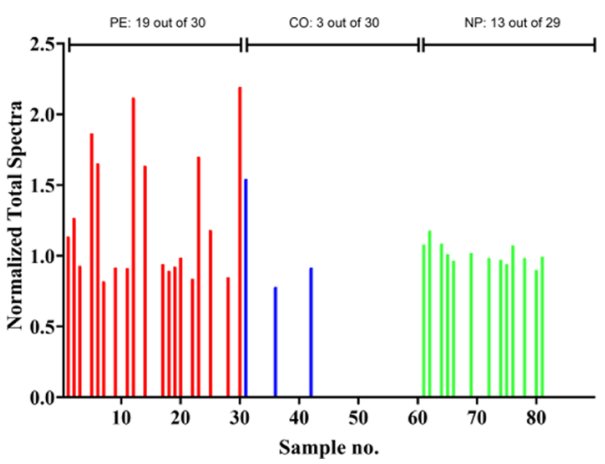

Figure 1. Normalized total spectra of MGP in the PE (red), CO (blue), and NP (green) group. The empty bars indicate that the MGP is too low or absent to be identified. Top: the number of identified MGP containing samples per group.

When comparing CSF proteins of normotensive pregnant women with nonpregnant women, we observed a total of 85 proteins that can be listed together by these two analyses as pregnancy-associated proteins in CSF (according to the literature). A higher number of proteins (7\%), mostly consisting of pregnancy-related proteins, was identified in CSF of normotensive pregnant women compared to nonpregnant women. Four significant differentially abundant pregnancy-related proteins were found when comparing preeclamptic women with normotensive pregnant women. Three of these were calcium-binding proteins.

In our previous study, ${ }^{18}$ we compared CSF of women with $\mathrm{PE}$ to CSF of normotensive pregnant women. In the present study, we added a group of nonpregnant women. This addition gives the possibility to study CSF pregnancy-related proteins and proteins specifically associated with $\mathrm{PE}$ and not necessarily related to pregnancy. In analogy to our previous study, ${ }^{18}$ peptides that belong to protein AMBP were significantly more abundant in preeclamptic women than in the normotensive pregnant women. However, this protein did not pass the criteria for significance in the present study. An exact similar trend for most proteins (24 out of 25 total) was observed, although most relevant proteins mentioned in our previous study lost their significance. This loss of significance may be due to the lower number of samples used per group in the present study. For that reason, it might show the same trend for upregulation and downregulation of these proteins.

Notably, after comparing the preeclamptic women with the normotensive pregnant women, more proteins (mainly extracellular) related to cellular movement processes were found to be upregulated in preeclamptic women (using the Ingenuity software tool). The significant differentially abundant proteins $\mathrm{CDH} 4, \mathrm{~F} 10, \mathrm{FN} 1, \mathrm{HSPG} 2$ (also known as Perlecan), MGP, and TAGLN (6 out of the 15 proteins identified in both analytical methods) found in this study are known to be calcium-binding proteins (Tables 3 and 4). ${ }^{19-24}$ MGP, using the Progenesis method and the Scaffold method, was elevated in PE (fold changes of 2.13 and 3.80, respectively). Although it is classified as a possible pregnancy-related protein, it had a low weighted score for literature mentioning pregnancy $(0.02$, Table 2$)$. MGP protein is part of the family of vitamin-K2-dependent GLA- $(\gamma$ carboxyglutamic acids) containing proteins, which have a high-affinity binding to calcium ions. It is secreted among smooth muscle cells and plays an active role in angiogenesis.

Using the two analytical methods Progenesis and Scaffold, 3 out of the 15 proteins (FMOD, MGP, and TAGLN) elevated in CSF of PE patients were associated with well-known pregnancy proteins. TAGLN is an actin-binding protein that belongs to the calponin family. Both proteins (TAGLN and MGP) are expressed in smooth muscle cells of developing vasculature and most likely contribute to vasculogenic dysfunction of PE. ${ }^{21,25,26}$ Similar to MGP and TAGLN, the differentially upregulated HSPG2 protein is also associated with vascularity. This extracellular matrix protein has an active role in vascular homeostasis. The FMOD protein, which was also found to be differentially upregulated in PE, may play a role in collagen fibrillogenesis. Assembly of extracellular matrix and regulation of TGF- $\beta$ activity are known processes of this glycoprotein. $^{27}$

The pregnancy-related proteins FN1, MGP, and TAGLN were significantly differentially abundant in $\mathrm{PE}$, as observed in both analyses. From these two analyses in which 15 differential abundant PE proteins were found together, 6 (CDH4, F10, FN1, HSPG2, MGP, TAGLN) have an affinity to bind calcium, as described above. The chance that a protein is related to calcium-binding proteins just by chance with both methods (Progenesis and Scaffold) is low. In five sets of 15 randomly taken proteins (from the total number of proteins identified $(n=817))$, we determined that $(12 \pm 6) \%$ were associated with calcium-binding (www.uniprot.org, searched for the term calcium-binding). Thus, we can conclude that the 6 calcium-binding proteins of the 15 proteins found is a significant finding. Most of the pregnancy-related proteins $(n=$ 66) analyzed with both Progenesis and Scaffold methods were not significantly different between preeclamptic women and

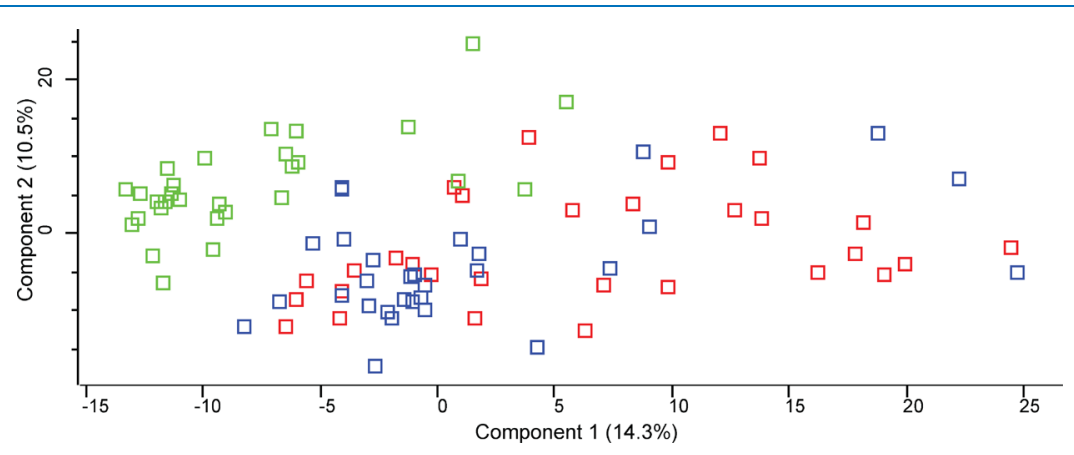

Figure 2. PCA analysis for all three groups (preeclampsia, normotensive pregnant, nonpregnant) analyzed. Variance in the first direction is $14.3 \%$, and that in the second dimension is $10.5 \%$. Groups indicated with red $=$ preeclamptic women, blue $=$ normotensive pregnant women, green $=$ nonpregnant women. 
normotensive pregnant women. Apart from the calciumbinding proteins CDH4, F10, FN1, HSPG2, MGP, and TAGLN found to be elevated in PE, it is tempting to speculate that more calcium-binding proteins might be related to $\mathrm{PE}$. For instance, Calcyclin (S100A6) and Galectin 13 (PP13) are both calcium-binding proteins that have been mentioned before to be involved in $\mathrm{PE}^{28,29}$ Calcium and magnesium are administered to PE women. The literature shows that the administration of calcium might have some beneficial effect, but is not a remedy. ${ }^{30-34}$

Although the total protein abundances (UV peak areas) were not significantly different between groups, the increase of CSF protein identifications in pregnancy indicates the possibility that the BBB permits passage of pregnancy-related proteins. In normal pregnancy in animal models, the permeability of the blood-brain barrier is described to be increased. $^{35,36}$ This change in permeability might also occur in humans. In an animal study investigating arterial reactivity, the permeability of the blood-brain barrier in PE was increased compared with normal pregnancy. ${ }^{11}$ The albumin ratio is a standardized biomarker value to determine the effect of potential BBB damage. ${ }^{37,38}$ In this study, we did not find any evidence of potential BBB damage in normotensive pregnant women or women with PE related to serum albumin levels. On the contrary, albumin levels were even significantly higher in CSF of nonpregnant women. The observation that albumin is even higher in nonpregnant women might be a homeostatic compensation mechanism for the influx of pregnancy-related proteins in CSF of pregnant women. A similar trend was observed for immunoglobulins (upregulated in nonpregnant women; Table S3).

The differences in the abundance of pregnancy-related proteins might be explained through changes in the brain that might occur during pregnancy. ${ }^{17}$ Apart from the fact that specific protein hormones change during pregnancy in CSF, research on relatively large numbers of pregnancy-associated proteins in CSF is just absent or partially available. It is of interest to study the precise functioning of these relatively large numbers of pregnancy proteins in the brain.

Second, it is of interest to further study mechanisms in which calcium and magnesium are incorporated in proteins associated with PE as a function of calcium and magnesium administration.

Strengths and Limitations of This Study. The strengths included the high-resolution mass spectrometry (MS) possibility of studying proteins and modifications in CSF proteins. Also, it is difficult to investigate molecular effects of pregnancy in the brain. Investigation of CSF proteins might be a new possibility to study the brain of pregnant women and preeclamptic women. Magnetic resonance imaging (MRI) can provide information about the brain of pregnant women. ${ }^{17}$ The possibility of investigating changes in the brain of women as a function of CSF proteins and by MRI may result in complementary information about women who suffer from $\mathrm{PE}$ and severe brain complications.

Mass spectrometry analyses can result in different outcomes depending on the analysis type used. If an analysis is focused on particular peptides or proteins, the overlap in the shotgun proteomic analysis is not high. This can be seen as a weakness and also as a strength because multiple analyses may complement each other as described above. Another weakness is the performed survey of interactions between calcium and magnesium concentrations and proteins as a function of magnesium administration. In the present study, cluster analysis of the group of preeclamptic women in which 24 out of the 30 patients were administered magnesium during sampling did not show any clustering compared to women with no magnesium administered. However, the number of samples is limited to conclude about any possible interactions between calcium and magnesium on protein level compared to the administration of magnesium.

\section{CONCLUSIONS}

We found differentially abundant proteins between preeclamptic women and normotensive pregnant women through both analysis methods used ( 15 proteins, of which 6 were calciumbinding). By comparing normotensive pregnant women with nonpregnant women, a total of 158 CSF proteins were found to be associated with pregnancy, from which 85 were mentioned as pregnancy-associated in the literature. To our knowledge, we showed for the first time that a $7 \%$ higher number of pregnancy-associated proteins were present in CSF of pregnant women. This $7 \%$ of proteins did not contribute to the top $10 \%$ of most abundant proteins found in CSF.

\section{MATERIAL AND METHODS}

Samples. A part of randomly selected CSF samples was used from the previous study to keep similar group size. ${ }^{18}$ Thirty out of 52 preeclamptic women, 30 out of 58 normotensive pregnant women, and an additional group of 29 nonpregnant women were obtained at two hospitals, the Erasmus MC (Rotterdam, The Netherlands) and Maasstad Hospital (Rotterdam, The Netherlands). After written consent, a CSF sample $(1 \mathrm{~mL})$ was obtained during the spinal anesthesia procedure before cesarean section in pregnant women. In nonpregnant women, this sample was obtained while performing spinal anesthesia for an elective surgical procedure. The needle used during the spinal puncture was a 25 or 27 Gauge atraumatic needle. The CSF sample was collected before the administration of anesthetics. Within $1 \mathrm{~h}$ after sampling, the CSF samples were centrifuged, and cells in the pellet were discarded. The CSF was aliquoted and stored immediately at $-20{ }^{\circ} \mathrm{C}$ and subsequently stored within $8 \mathrm{~h}$ at $-80{ }^{\circ} \mathrm{C}$.

Approval for the study was given by the Ethics Board (MEC 2007-086 and MEC 2015-457).

Shotgun Proteomics. The samples were processed using CSF digestion conform to the mass spectrometry (MS) measurement protocol as previously described by van den Berg et al., ${ }^{18}$ with adjustments in Orbitrap instrument, LC-gradient, and higher sample volume $(3 \mu \mathrm{L})$. The shotgun proteomic data have been deposited to the ProteomeXchange Consortium via the PRIDE $^{39}$ partner repository with the dataset identifier PXD017862 and 10.6019/PXD017862.

The samples were enzymatically digested with trypsin and subsequently measured with a nano-LC-Orbitrap MS/MS mass spectrometry system (Ultimate 3000 HPLC, Thermo Fisher Scientific, Germering, Germany; Orbitrap Lumos, Thermo Fisher Scientific, San Jose, CA). Three-microliter digest was loaded on a C18 trap column (C18 PepMap, 300 $\mu \mathrm{m}$ inner diameter (ID) $\times 5 \mathrm{~mm}, 5 \mu \mathrm{m}$ particle size, $100 \AA$ pore size; Thermo Fisher Scientific, The Netherlands) and desalted for $10 \mathrm{~min}$ using a flow rate of $20 \mu \mathrm{L} / \mathrm{min} 0.1 \%$ TFA. The trap column was switched online with the analytical column (PepMap C18, $75 \mu \mathrm{m}$ ID $\times 250 \mathrm{~mm}, 2 \mu \mathrm{m}$ particle, 
and $100 \AA$ pore size; Dionex, The Netherlands) and peptides were eluted with the following binary (A and B) gradient: 4$38 \%$ solvent B in $90 \mathrm{~min}$, whereby solvent A consists of $2 \%$ acetonitrile and $0.1 \%$ formic in water and solvent B consists of $80 \%$ acetonitrile and $0.08 \%$ formic acid in water. The column flow rate was set to $300 \mathrm{~nL} / \mathrm{min}$.

A data-dependent acquisition method was used for MS detection: a high-resolution survey scan from 375 to $1500 \mathrm{Th}$. was performed in the Orbitrap (value of target of automatic gain control (AGC) 400000 ) and a resolution 120000 at 400 $\mathrm{m} / z$; lock mass was set to $445.12003 \mathrm{u}$ (protonated $\left.\left(\mathrm{Si}\left(\mathrm{CH}_{3}\right)_{2} \mathrm{O}\right)_{6}\right)$. Based on this survey scan, the most intense ions were consecutively isolated (AGC target set to $10^{4}$ ions) and fragmented by collision-activated dissociation (CAD) applying 35\% normalized collision energy in the linear ion trap until a duty cycle time of $3 \mathrm{~s}$ was reached (top speed mode). After precursors were selected for MS/MS, they were excluded for further MS/MS spectra for $60 \mathrm{~s}$.

Data Analysis. Data were analyzed in two ways: (1) for peptide and subsequent protein identifications, the raw data were processed and analyzed, as previously described ${ }^{18}$ using Progenesis software (version 4.0, Nonlinear Dynamics, Newcastle-upon-Tyne, U.K.) ("Progenesis method") and (2) using Scaffold software (Proteome Software, version 4.7.2, Proteome Software, Inc., Portland, OR) in which just proteins identified by Mascot were loaded and analyzed ("Scaffold method").

The identification of proteins through the Progenesis method is a criteria-based analysis on peptide level. ${ }^{18}$ The criteria were defined as: (1) $50 \%$ or more of the peptides of the protein must have a low $p$-value $(p<0.05)$; (2) $30 \%$ of the peptides of the protein must have a very low $p$-value $(p<$ 0.01 ); and (3) $75 \%$ or more of the peptides of the protein must be altered in the same direction between the groups. The statistical background level was determined by performing a permutation test using all samples randomly with an identical analysis. The permutation test was repeated 1000 times, saving the resulting thresholds with $\mathrm{SD}^{18}$ for determining the significance value received from the actual experiment.

The identification of proteins through the Scaffold method is directly based on protein level. The Scaffold method was performed based on quantitative normalized spectral counts to determine the abundances of identified proteins. The significance of protein abundances between groups was determined by a $t$-test corrected for multiple testing by Benjamini-Hochberg analysis. This significance was calculated in the Scaffold software program. A value below the false discovery rate, as calculated by Benjamini-Hochberg analysis, was considered to be significantly different. The abundance of each protein is determined by calculating the fold change. Zero counts were converted to 0.125 to enable log calculations.

In the present study, first, we searched for pregnancy-related proteins by comparing the normotensive control pregnant group (healthy pregnant) with nonpregnant women, using the Progenesis and Scaffold methods. Proteins were considered to be pregnancy-related if they were significantly up- or downregulated in the normotensive pregnant group compared to the nonpregnant group. Subsequently, each protein was searched in the Scopus citation database to survey the relation with pregnancy, and each protein was sorted according to their weighted score. The number of pregnancy hits was counted by searching the particular protein name combined with the word "pregnancy" that was found either in the abstract or in the title section of the selected publications (pregnancy in the protein name itself was excluded in this literature search). The score was calculated by the following formula: total number of pregnancy hits * [ total number of pregnancy hits/total number of hits] for each protein separately. We used this formula to prevent that a protein has a high percentage score if the total number of hits and number of pregnancy hits were both relatively low. Second, we compared the preeclamptic women with the normotensive pregnant women by both Progenesis and Scaffold methods.

Principal component analysis was performed for comparison within the three groups, preeclamptic, normotensive pregnant, and nonpregnant women. The Ingenuity Pathway Analysis (IPA; www.qiagen.com) tool was used to get insight into the functionality of proteins in CSF in women during pregnancy and in women with PE. A flowchart of the various methods is shown in Figure 3.

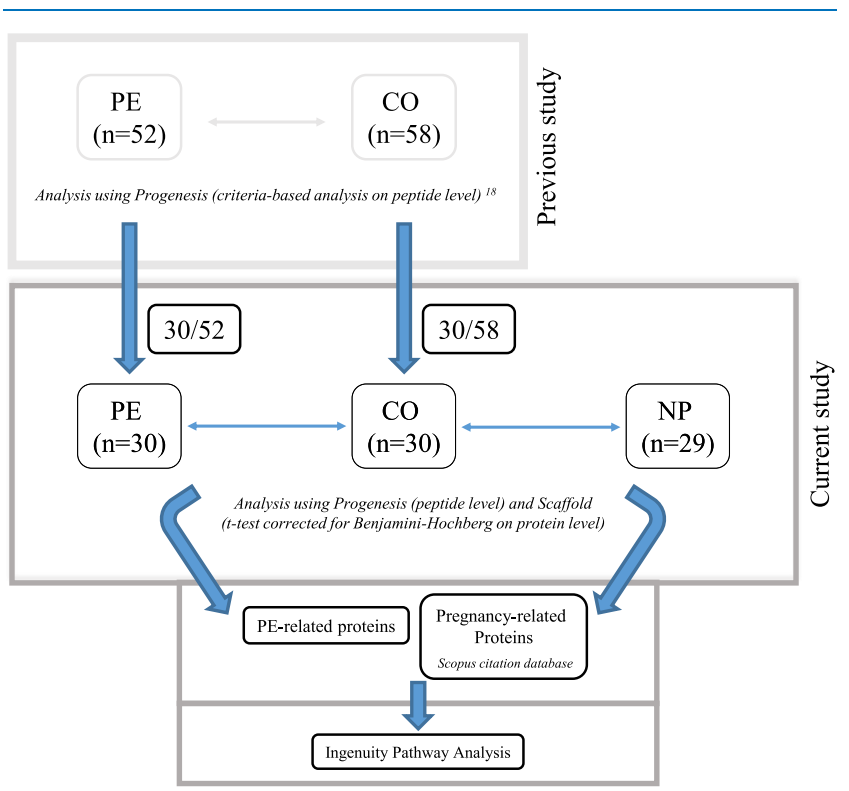

Figure 3. Flowchart of data analysis. Progenesis and Scaffold methods for the group comparisons of women with PE (preeclampsia, $n=30$ ), $\mathrm{CO}$ (normotensive pregnant women, $n=30$ ), and NP (nonpregnant women, $n=29$ ). The extra group (NP) containing 29 subjects was added to a part of the samples used in the previous study, ${ }^{18}$ which included 52 (current $n=30$ ) preeclamptic women and 58 (current $n$ $=30$ ) normotensive pregnant women. The number of subjects in PE and $\mathrm{CO}$ was reduced to keep comparable numbers in each group.

UV traces (peak areas (mAU*min)) from liquid chromatography-mass spectrometry (LC-MS) measurements related to preeclamptic, normotensive pregnant, and nonpregnant women were compared to each other to determine the total protein content in a fixed volume of $3 \mu \mathrm{L} \mathrm{CSF}$. An analysis of variance (ANOVA) $t$-test was performed to determine whether a difference exists between protein peak areas of the groups mentioned above.

\section{ASSOCIATED CONTENT}

\section{Supporting Information}

The Supporting Information is available free of charge at https://pubs.acs.org/doi/10.1021/acsomega.0c03910.

Clinical characteristics (Table S1); differently abundant proteins in CSF of normotensive pregnant women and nonpregnant women as analyzed by the Progenesis 
method (Table S2); differently abundant proteins in CSF of normotensive pregnant women and nonpregnant women as analyzed by the Scaffold method (Table S3); and comparison of proteins found in our previous study with the current study (Table S4) (PDF)

\section{AUTHOR INFORMATION}

\section{Corresponding Author}

Theo M. Luider - Laboratory of Neuro-Oncology/Clinical \& Cancer Proteomics, Department of Neurology, Erasmus University Medical Center, Rotterdam 3015 CN, The Netherlands; 이이.org/0000-0003-1962-561X; Phone: (+31) 10 7038069; Email: t.luider@erasmusmc.nl

\section{Authors}

Coşkun Güzel - Laboratory of Neuro-Oncology/Clinical \& Cancer Proteomics, Department of Neurology, Erasmus University Medical Center, Rotterdam 3015 CN, The Netherlands; 이이.org/0000-0002-9849-9174

Caroline B. van den Berg - Department of Obstetrics and Gynecology, Erasmus University Medical Center, Rotterdam 3015 GD, The Netherlands

Seppe Koopman - Department of Anesthesiology, Maasstad Hospital, Rotterdam 3079 DZ, The Netherlands

Robbert Jan van Krugten - Department of Anesthesiology, Maasstad Hospital, Rotterdam 3079 DZ, The Netherlands

Marcel Stoop - Laboratory of Neuro-Oncology/Clinical \& Cancer Proteomics, Department of Neurology, Erasmus University Medical Center, Rotterdam 3015 CN, The Netherlands

Christoph Stingl - Laboratory of Neuro-Oncology/Clinical \& Cancer Proteomics, Department of Neurology, Erasmus University Medical Center, Rotterdam 3015 CN, The Netherlands

Johannes J. Duvekot - Department of Obstetrics and Gynecology, Erasmus University Medical Center, Rotterdam 3015 GD, The Netherlands

Complete contact information is available at: https://pubs.acs.org/10.1021/acsomega.0c03910

\section{Funding}

This research did not receive any specific grant from funding agencies in the public, commercial, or not-for-profit sectors.

\section{Notes}

The authors declare no competing financial interest.

\section{ACKNOWLEDGMENTS}

The authors gratefully thank the participants for donating their csf samples.

\section{REFERENCES}

(1) Steegers, E. A.; von Dadelszen, P.; Duvekot, J. J.; Pijnenborg, R. Pre-eclampsia. Lancet 2010, 376, 631-644.

(2) Aukes, A. M.; de Groot, J. C.; Aarnoudse, J. G.; Zeeman, G. G. Brain lesions several years after eclampsia. Am. J. Obstet. Gynecol. 2009, 200, 504.e1-504.e5.

(3) Aukes, A. M.; De Groot, J. C.; Wiegman, M. J.; Aarnoudse, J. G.; Sanwikarja, G. S.; Zeeman, G. G. Long-term cerebral imaging after pre-eclampsia. BJOG 2012, 119, 1117-1122.

(4) Johnson, A. C.; Nagle, K. J.; Tremble, S. M.; Cipolla, M. J. The Contribution of Normal Pregnancy to Eclampsia. PLoS One 2015, 10, No. e0133953.
(5) Kimelberg, H. K. Water homeostasis in the brain: basic concepts. Neuroscience 2004, 129, 851-860.

(6) Hawkins, B. T.; Davis, T. P. The blood-brain barrier/ neurovascular unit in health and disease. Pharmacol. Rev. 2005, 57, 173-185.

(7) Rubin, L. L.; Staddon, J. M. The cell biology of the blood-brain barrier. Annu. Rev. Neurosci. 1999, 22, 11-28.

(8) Goasdoué, K.; Miller, S. M.; Colditz, P. B.; Bjorkman, S. T. Review: The blood-brain barrier; protecting the developing fetal brain. Placenta 2017, 54, 111-116.

(9) Zeeman, G. G. Neurologic complications of pre-eclampsia. Semin. Perinatol. 2009, 33, 166-172.

(10) Varatharaj, A.; Galea, I. The blood-brain barrier in systemic inflammation. Brain, Behav., Immun. 2017, 60, 1-12.

(11) Amburgey, O. A.; Chapman, A. C.; May, V.; Bernstein, I. M.; Cipolla, M. J. Plasma from preeclamptic women increases bloodbrain barrier permeability: role of vascular endothelial growth factor signaling. Hypertension 2010, 56, 1003-1008.

(12) Burwick, R. M.; Togioka, B. M.; Speranza, R. J.; Gaffney, J. E.; Roberts, V. H. J.; Frias, A. E.; Rincon, M. Assessment of blood-brain barrier integrity and neuroinflammation in preeclampsia. Am. J. Obstet. Gynecol. 2019, 221, 269.e1-269.e8.

(13) Spitzer, P.; Klafki, H. W.; Blennow, K.; Buee, L.; Esselmann, H.; Herruka, S. K.; Jimenez, C.; Klivenyi, P.; Lewczuk, P.; Maler, J. M.; Markus, K.; Meyer, H. E.; Morris, C.; Muller, T.; Otto, M.; Parnetti, L.; Soininen, H.; Schraen, S.; Teunissen, C.; Vecsei, L.; Zetterberg, H.; Wiltfang, J. cNEUPRO: Novel Biomarkers for Neurodegenerative Diseases. Int. J. Alzheimers Dis. 2010, 2010, No. 548145.

(14) Singh, V.; Hintzen, R. Q.; Luider, T. M.; Stoop, M. P. Proteomics technologies for biomarker discovery in multiple sclerosis. J. Neuroimmunol. 2012, 248, 40-47.

(15) Staff, A. C.; Dechend, R. Preeclampsia: What Does the Brain Tell Us? Can We Blame the Eclampsia Risk on a Malperfused Placenta? Hypertension 2018, 72, 65-67.

(16) Wang, Y.; Sun, S.; Zhu, J.; Cui, L.; Zhang, H. L. Biomarkers of Guillain-Barre Syndrome: Some Recent Progress, More Still to Be Explored. Mediators Inflammation 2015, 2015, No. 564098.

(17) Barba-Müller, E.; Craddock, S.; Carmona, S.; Hoekzema, E. Brain plasticity in pregnancy and the postpartum period: links to maternal caregiving and mental health. Arch. Womens Ment. Health 2019, 22, 289-299.

(18) van den Berg, C. B.; Duvekot, J. J.; Güzel, C.; Hansson, S. R.; de Leeuw, T. G.; Steegers, E. A.; Versendaal, J.; Luider, T. M.; Stoop, M. P. Elevated levels of protein AMBP in cerebrospinal fluid of women with preeclampsia compared to normotensive pregnant women. Proteomics: Clin. Appl. 2017, 11, No. 1600082.

(19) Ma, H.; Zhang, B. L.; Liu, B. Y.; Shi, S.; Gao, D. Y.; Zhang, T. C.; Shi, H. J.; Li, Z.; Shum, W. W. Vitamin K2-Dependent GGCX and MGP Are Required for Homeostatic Calcium Regulation of Sperm Maturation. iScience 2019, 14, 210-225.

(20) Farach-Carson, M. C.; Carson, D. D. Perlecan-a multifunctional extracellular proteoglycan scaffold. Glycobiology 2007, 17, 897905.

(21) Assinder, S. J.; Stanton, J. A.; Prasad, P. D. Transgelin: an actinbinding protein and tumour suppressor. Int. J. Biochem. Cell Biol. 2009, 41, 482-486.

(22) Khamchun, S.; Sueksakit, K.; Chaiyarit, S.; Thongboonkerd, V. Modulatory effects of fibronectin on calcium oxalate crystallization, growth, aggregation, adhesion on renal tubular cells, and invasion through extracellular matrix. J. Biol. Inorg. Chem. 2019, 24, 235-246.

(23) Li, Z.; Su, D.; Ying, L.; Yu, G.; Mao, W. Study on expression of $\mathrm{CDH} 4$ in lung cancer. World J. Surg. Oncol. 2017, 15, 26.

(24) Xu, X.; Chen, J.; Zhang, L.; Wang, S.; Shen, D.; Liu, Q. Calcium ion-induced stabilization and refolding of agkisacutacin from Agkistrodon acutus venom studied by fluorescent spectroscopy. $J$. Fluoresc. 2007, 17, 215-221.

(25) Varberg, K. M.; Garretson, R. O.; Blue, E. K.; Chu, C.; Gohn, C. R.; Tu, W.; Haneline, L. S. Transgelin induces dysfunction of fetal 
endothelial colony-forming cells from gestational diabetic pregnancies. Am. J. Physiol.: Cell Physiol. 2018, 315, C502-C515.

(26) Gheorghe, S. R.; Craciun, A. M. Matrix Gla protein in tumoral pathology. Clujul Med. 2016, 89, 319-321.

(27) Pourhanifeh, M. H.; Mohammadi, R.; Noruzi, S.; Hosseini, S. A.; Fanoudi, S.; Mohamadi, Y.; Hashemzehi, M.; Asemi, Z.; Mirzaei, H. R.; Salarinia, R.; Mirzaei, H. The role of fibromodulin in cancer pathogenesis: implications for diagnosis and therapy. Cancer Cell Int. 2019, 19, 157.

(28) Güzel, C.; van den Berg, C. B.; Duvekot, J. J.; Stingl, C.; van den Bosch, T. P. P.; van der Weiden, M.; Steegers, E. A. P.; SteegersTheunissen, R. P. M.; Luider, T. M. Quantification of Calcyclin and Heat Shock Protein 90 in Sera from Women with and without Preeclampsia by Mass Spectrometry. Proteomics: Clin. Appl. 2019, 13, No. e1800181.

(29) Sammar, M.; Drobnjak, T.; Mandala, M.; Gizurarson, S.; Huppertz, B.; Meiri, H. Galectin 13 (PP13) Facilitates Remodeling and Structural Stabilization of Maternal Vessels during Pregnancy. Int. J. Mol. Sci. 2019, 20, No. 3192.

(30) Ephraim, R. K.; Osakunor, D. N.; Denkyira, S. W.; Eshun, H.; Amoah, S.; Anto, E. O. Serum calcium and magnesium levels in women presenting with pre-eclampsia and pregnancy-induced hypertension: a case-control study in the Cape Coast metropolis, Ghana. BMC Pregnancy Childbirth 2014, 14, 390.

(31) Levine, R. J.; Hauth, J. C.; Curet, L. B.; Sibai, B. M.; Catalano, P. M.; Morris, C. D.; DerSimonian, R.; Esterlitz, J. R.; Raymond, E. G.; Bild, D. E.; Clemens, J. D.; Cutler, J. A. Trial of calcium to prevent preeclampsia. N. Engl. J. Med. 1997, 337, 69-76.

(32) Sun, X.; Li, H.; He, X.; Li, M.; Yan, P.; Xun, Y.; Lu, C.; Yang, $\mathrm{K}$; Z Zhang, $\mathrm{X}$. The association between calcium supplement and preeclampsia and gestational hypertension: a systematic review and meta-analysis of randomized trials. Hypertens. Pregnancy 2019, 38, 129-139.

(33) Hofmeyr, G. J.; Manyame, S.; Medley, N.; Williams, M. J. Calcium supplementation commencing before or early in pregnancy, for preventing hypertensive disorders of pregnancy. Cochrane Database Syst. Rev. 2019, 9, No. CD011192.

(34) ACOG Practice Bulletin No. 202. Gestational Hypertension and Preeclampsia. Obstet. Gynecol. 2019, 133, e1-e25.

(35) Miller, E. C. Preeclampsia and Cerebrovascular Disease. Hypertension 2019, 74, 5-13.

(36) Cipolla, M. J.; Sweet, J. G.; Chan, S. L. Cerebral vascular adaptation to pregnancy and its role in the neurological complications of eclampsia. J. Appl. Physiol. 2011, 110, 329-339.

(37) Tibbling, G.; Link, H.; Ohman, S. Principles of albumin and IgG analyses in neurological disorders. I. Establishment of reference values. Scand. J. Clin. Lab. Invest. 1977, 37, 385-390.

(38) Kirch, D. G.; Alexander, R. C.; Suddath, R. L.; Papadopoulos, N. M.; Kaufmann, C. A.; Daniel, D. G.; Wyatt, R. J. Blood-CSF barrier permeability and central nervous system immunoglobulin $G$ in schizophrenia. J. Neural Transm.: Gen. Sect. 1992, 89, 219-232.

(39) Perez-Riverol, Y.; Csordas, A.; Bai, J.; Bernal-Llinares, M.; Hewapathirana, S.; Kundu, D. J.; Inuganti, A.; Griss, J.; Mayer, G.; Eisenacher, M.; Perez, E.; Uszkoreit, J.; Pfeuffer, J.; Sachsenberg, T.; Yilmaz, S.; Tiwary, S.; Cox, J.; Audain, E.; Walzer, M.; Jarnuczak, A. F.; Ternent, T.; Brazma, A.; Vizcaino, J. A. The PRIDE database and related tools and resources in 2019: improving support for quantification data. Nucleic Acids Res. 2019, 47, D442-D450. 